\section{Prevalência e fatores associados à dor lombar em escolares}

\author{
Prevalence of lower back pain and \\ associated factors in students
}

\author{
1 Universidade do Sagrado \\ Coração, Bauru, Brasil. \\ Correspondência \\ A. De Vitta \\ Universidade do Sagrado \\ Coração. \\ Rua Irmã Arminda 10-50, \\ Bauru, SP 17011-160, Brasil. \\ albvitta@yahoo.com.br
}

\begin{abstract}
The objective was to determine the prevalence of lower back pain in adolescents and its relationship to sports and sedentary activities. We conducted a cross-sectional study of 5 th to 8 th-grade students $(n=1,236)$ in Bauru, São Paulo State, Brazil. We used a structured protocol and the Nordic questionnaire for musculoskeletal symptoms. The analysis was performed using a descriptive approach and bivariate and multivariate binary logistic regression. Prevalence of lower back pain was $19.5 \%$ (7\% in boys and $12.5 \%$ in girls), with statistically significant differences according to gender $(p<0.00001)$, age $(p=0.0057)$, and sports $(p=0.0001)$. Bivariate and multivariate analyses showed independent associations between lower back pain and female gender, time watching TV, and sports. Lower back pain in schoolchildren can persist as chronic pain in adults. Understanding the relationships between variables should provide useful measures for maintaining, improving, and promoting students' wellbeing.
\end{abstract}

Low Back Pain; Students; Adolescent; Risk Factors; Prevalence

\author{
Alberto De Vitta 1 \\ Mariana Gonçalez Martinez 1 \\ Nathália Toledo Piza 1 \\ Sandra Fiorelli de Almeida Penteado Simeão 1 \\ Nathália Pascucci Ferreira 1
}

\section{Introdução}

A dor lombar tornou-se um grave problema de saúde pública, pois possui alta incidência na população economicamente ativa, em adolescentes e crianças 1 . A prevalência de dores lombares em crianças de 9 a 10 anos é similar a da população adulta ${ }^{2}$. Em um estudo longitudinal com adolescentes de 12 a 15 anos, foi observado que a incidência anual de sintomas musculoesqueléticos na coluna vertebral foi de $21,5 \%{ }^{3}$. Em outro estudo com 1.389 escolares dinamarqueses de 13 a 16 anos, foi constatado que $19,4 \%$ apresentaram dores na coluna lombar 4 .

Ainda na Dinamarca, outra pesquisa com escolares apontou que a incidência de dor lombar foi de $33 \%$ em crianças de 8 a 10 anos e $47 \%$ em adolescentes de 14 a 16 anos 5 . Em estudo similar que observou 254 meninas na faixa etária de 8 a 16 anos, notou-se que $31,4 \%$ apresentaram dor na coluna lombar 6 .

Os fatores de risco para o desenvolvimento de dores lombares são multifatoriais, tais como o gênero, antropometria (altura e peso corporal), diminuição da flexibilidade e mobilidade dos músculos, hipermobilidade, obesidade, psicossociais, participação em esportes de competição; o peso, tipo e modo de carregar a mochila; hábitos posturais incorretos; atividade ocupacional após o período escolar; atividades sedentárias (tempo na frente da TV e no computador e/ ou $v i$ deogame); nível de atividade física e tabagismo ? 
De acordo com alguns estudos, as atividades sedentárias (tempo na frente da TV e no computador e/ou videogame) são fatores associados a dores lombares. Adolescentes com maior prevalência de dores na coluna lombar são os que passam maior tempo na frente da TV 8 . Crianças de nove anos que assistem à televisão ou jogam videogame por mais de duas horas ao dia apresentaram mais dores na coluna lombar 9 .

Em relação à participação em esportes de competição, estudos concluíram que essa prática é um importante fator de risco para dores na coluna lombar em escolares 10,11.

A relevância de estudos sobre a prevalência de dores lombares em escolares reside no fato de que essas patologias geram consequências sociais e econômicas, tanto para o Estado como para os indivíduos. Para o sujeito, significa a perda da qualidade de vida, e, para o Estado, as despesas com tratamento e reabilitação.

Dessa forma, considerando os estudantes de 5a a $8 \underline{a}$ séries do Ensino Fundamental municipal da cidade de Bauru, São Paulo, Brasil, o objetivo deste trabalho foi verificar a prevalência de dor lombar em adolescentes e sua relação com a prática de esportes de competição e atividades sedentárias (tempo na frente da TV e no computador e/ou videogame).

\section{Materiais e métodos}

Foi realizado um estudo epidemiológico observacional, transversal, no ano letivo de 2007, cuja variável dependente foi a dor na coluna lombar, e as independentes foram a prática de esportes de competição e atividades sedentárias (tempo na frente da TV e no computador e/ou videogame).

A quantidade de alunos matriculados no ensino fundamental, no ano de 2007, na cidade de Bauru, foi obtida na Diretoria de Ensino dessa cidade, órgão ligado à Secretaria Estadual de Educação. De acordo com esse órgão, havia 26.067 estudantes, sendo 14.985 (57,5\%) matriculados na rede estadual, $9.750(37,4 \%)$ em escolas particulares e $1.332(5,1 \%)$ na municipal.

Considerando esses percentuais, foram sujeitos da pesquisa todos os escolares matriculados no ensino fundamental (5a a $8 \underline{a}$ séries) de todas as escolas municipais de Bauru (5 unidades). Tal escolha deveu-se à disponibilidade de acesso à rede municipal devido a um convênio firmado entre a Universidade do Sagrado Coração e a Secretaria Municipal de Educação de Bauru. Essa situação não se repetiu nas escolas particulares e estaduais.

A Secretaria Municipal de Educação de Bauru emitiu uma carta de autorização. Os pais dos alunos foram informados sobre todas as etapas do estudo, o caráter voluntário da participação, a possibilidade de seu filho abandonar a pesquisa a qualquer momento e o direito ao sigilo dos dados individuais e assinaram o termo de consentimento. O projeto foi aprovado pelo Comitê de Ética em Pesquisa da Universidade do Sagrado Coração (processo no ${ }^{\circ}$ 119/06).

Os pesquisadores realizaram a coleta de dados no período de fevereiro a maio de 2007 , contando com a colaboração das professoras de cada classe, que enviavam um estudante de cada vez para responder o questionário. A cada aluno, eram explicados os objetivos do estudo, e, em seguida, o pesquisador realizava a entrevista estruturada, questão por questão, sendo que cada abordagem individual teve um tempo despendido de, aproximadamente, vinte minutos.

As variáveis independentes foram coletadas por meio do questionário 4 , composto das seguintes questões: idade (11, 12, 13 e 14 anos); sexo; "Você pratica modalidade esportiva de competição fora da escola?” (sim/não); “Em uma semana normal de aula, você assiste à TV?” (sim/ não); "Em uma semana normal de aula, quantas vezes você assiste à TV?” (uma vez, duas vezes, três vezes, quatro vezes, cinco vezes ou mais por semana); "Em um dia normal de aula, quantas horas você assiste à TV?” (menos de uma hora, duas horas, três horas, quatro horas, cinco horas ou mais por dia); "Em uma semana normal de aula, quantas vezes usa o computador ou videogame em casa?" (uma vez, duas vezes, três vezes, quatro vezes, cinco vezes ou mais por semana); "Em um dia normal de aula, quantas horas usa o computador ou videogame em casa?” (menos de uma hora, duas horas, três horas, quatro horas, cinco horas ou mais por dia).

As variáveis "número de vezes na semana e quantidade de horas ao dia que assiste à TV" e "número de vezes na semana e quantidade de horas ao dia que utiliza o computador" foram geradas a partir das uniões das variáveis "número de vezes que assiste à TV na semana" e "quantidade de horas que assiste à TV ao dia" e "número de vezes que utiliza o computador na semana" e "quantidade de horas que utiliza o computador ao dia”, respectivamente. O motivo de tal junção se deve à associação entre as mesmas quanto ao aspecto da presença ou não da dor. As variáveis foram categorizadas da seguinte forma: até 2 vezes e até 2 horas; até 2 vezes e acima de 2 horas; mais de 2 vezes e até 2 horas; mais de 2 vezes e acima de 2 horas.

A variável dependente - dor na coluna lombar - foi observada por meio do questionário nórdico, adaptado para a cultura brasileira 12 . A dor lombar foi definida como dor ou desconforto 
nos últimos 12 meses não relacionada à trauma ou à dor menstrual. No momento da entrevista, foi realizada a seguinte pergunta aos escolares: "Você teve dor na coluna lombar (parte inferior das costas) no último ano?”. Para maior especificidade sobre o local da dor, além do questionamento verbal, foi apresentada uma imagem das regiões da coluna vertebral, em diferentes cores, para que identificassem a região lombar. Esse tipo de ferramenta é válido e confiável para medir dor em escolares, pois os torna capazes de ser muito específicos na localização da dor 13 .

Considerando a multiplicidade das variáveis investigadas, o amplo universo de escolas e alunos, o instrumento de investigação e as características da equipe, foi realizado (abril de 2006), previamente, um estudo piloto com 35 alunos da quinta série, com a finalidade de averiguar a exequibilidade do projeto (evidenciando suas dificuldades a fim de minimizá-las) e promover o treinamento dos pesquisadores, reduzindo a possibilidade de tendenciosidade na coleta de dados. A linguagem simples dos questionários facilitou sua utilização em crianças e adolescentes.

Os dados obtidos foram introduzidos em um banco de dados e submetidos ao programa estatístico SPSS, versão 10.0 (SPSS Inc., Chicago, Estados Unidos). A análise foi realizada mediante uma abordagem descritiva e outra analítica. $\mathrm{Na}$ abordagem descritiva, foram feitas as distribuições de frequências absoluta e relativa para variáveis categóricas, e média e desvio-padrão, para as variáveis contínuas. Na abordagem analítica, foram realizadas uma análise bivariada por meio do teste de Pearson e, em seguida, uma análise multivariada por regressão logística binária, utilizando análise hierarquizada. O método de introdução das variáveis nos modelos adotados foi o backward stepwise. Considerou-se um nível de significância de 5\% e intervalo de 95\% de confiança (IC95\%), com cálculo dos odds ratios ajustados 14,15 .

\section{Resultados}

A população total de escolares matriculados, no ano de 2007, nas salas de 5a a 8 a séries de todas as escolas municipais de Bauru, totalizando 5 unidades, era de 1.332. Desse total, 1.236 responderam o questionário, e 96 se recusaram a participar do estudo, representando 7,2\% de perdas.

Considerando os dados acima, dos 1.236 escolares que responderam o questionário, 596 eram do sexo masculino, e 640, do feminino. Desses, 340 cursavam a $5 \underline{a}$ série, sendo 166 do sexo masculino e 174 do feminino; 323 a 6a série, sendo 145 masculinos e 178 femininos; 312 estavam matriculados na 7a árie, sendo 148 do sexo masculino e 164 do feminino, e 261, matriculados na 8a série, sendo 137 do sexo masculino e 124 do sexo feminino.

Na Tabela 1, nota-se a distribuição dos estudantes por faixa etária, participação na aula de educação física, prática de esportes e atividades sedentárias, segundo o sexo. Em relação à idade, observa-se que há maior porcentagem de meninos com 14 anos (34,5\%) e de meninas com 13 $\operatorname{anos}(28,2 \%)$.

Quanto à participação na aula de educação física, notou-se que 95,5\% dos meninos e 92,9\% das meninas realizam essa atividade escolar, enquanto que $46,8 \%$ dos meninos e $45,8 \%$ das meninas frequentam alguma modalidade esportiva fora da escola.

De acordo com as respostas dos estudantes, verificou-se que os esportes mais praticados fora do ambiente escolar pelo sexo feminino são natação $(20,3 \%)$, futebol $(10,9 \%)$ e basquetebol $(10,4 \%)$ e, pelo masculino, são o futebol (15\%) e voleibol (14,5\%).

Quanto às atividades sedentárias (tempo na frente da TV e no computador e/ou videogame), verificou-se que 593 meninos $(99,4 \%)$ e 635 meninas $(99,2 \%)$ assistem à TV. Em relação ao número de vezes na semana e quantidade de horas ao dia diante da tela da TV, $66,8 \%$ dos adolescentes e 70,9\% das adolescentes passam mais de 2 vezes por semana e acima de 2 horas por dia utilizando esse equipamento.

No que diz respeito ao computador, 481 meninos $(80,7 \%)$ e 439 meninas $(68,3 \%)$ utilizam o equipamento, e, quanto ao número de vezes na semana e quantidade de horas ao dia de uso do mesmo, 38,7\% dos meninos relataram que usam mais de duas vezes por semana e acima de 2 horas por dia, enquanto que $55,5 \%$ das meninas utilizam o computador/videogame até duas vezes por semana e até duas horas por dia.

A Tabela 2 indica que a prevalência de dor lombar nos escolares foi de $19,5 \%$, sendo $35,6 \%$ nos meninos e $64,4 \%$ nas meninas, com diferença estatisticamente significante entre os gêneros ( $\mathrm{p}=0,0000014)$, assim como entre a faixa etária $(\mathrm{p}=0,0057)$ e a prática de esportes $(\mathrm{p}=0,0001)$.

A associação entre dor lombar e as atividades sedentárias mostrou significância estatística para os fatores de risco: horas na frente da TV ( $\mathrm{p}=$ 0,0001) e número de vezes na semana associado à quantidade de horas ao dia diante da tela da TV $(\mathrm{p}=0,0009)$ (Tabela 3).

Na análise de regressão logística multivariada, foi verificado, na Tabela 4, que os fatores associados com os sintomas na coluna lombar foram o gênero feminino, quantidade de horas que assiste à TV ao dia e a prática de esportes. 
Distribuição das frequências absoluta e relativa da faixa etária, participação na aula de educação física, prática de esportes fora da escola e atividades sedentárias, segundo o sexo.

\begin{tabular}{|c|c|c|c|c|c|c|}
\hline \multirow[t]{3}{*}{ Variável/Resposta } & \multicolumn{4}{|c|}{ Sexo } & \multirow{2}{*}{\multicolumn{2}{|c|}{$\begin{array}{c}\text { Total } \\
(n=1.236)\end{array}$}} \\
\hline & \multicolumn{2}{|c|}{ Masculino $(n=596)$} & \multicolumn{2}{|c|}{ Feminino $(n=640)$} & & \\
\hline & $\mathrm{n}$ & $\%$ & n & $\%$ & $\mathbf{n}$ & $\%$ \\
\hline \multicolumn{7}{|l|}{ Faixa etária (anos) } \\
\hline 11 & 99 & 16,6 & 128 & 20,0 & 227 & 18,4 \\
\hline 12 & 129 & 21,6 & 162 & 25,3 & 291 & 23,5 \\
\hline 13 & 162 & 27,1 & 180 & 28,2 & 342 & 27,6 \\
\hline 14 & 206 & 34,5 & 170 & 26,5 & 376 & 30,5 \\
\hline \multicolumn{7}{|l|}{ Participação na aula de Educação Física } \\
\hline Não & 27 & 4,5 & 45 & 7,1 & 72 & 5,8 \\
\hline $\operatorname{Sim}$ & 569 & 95,5 & 595 & 92,9 & 1.164 & 94,2 \\
\hline \multicolumn{7}{|l|}{ Prática de esportes fora da escola } \\
\hline Não & 317 & 53,2 & 347 & 54,2 & 664 & 53,7 \\
\hline Sim & 279 & 46,8 & 293 & 45,8 & 572 & 46,3 \\
\hline \multicolumn{7}{|c|}{ Número de vezes que assiste à TV na semana } \\
\hline Até 2 & 63 & 10,6 & 54 & 8,5 & 117 & 9,5 \\
\hline Mais de 2 & 530 & 89,4 & 581 & 91,5 & 1.111 & 90,5 \\
\hline \multicolumn{7}{|c|}{ Quantidade de horas que assiste à TV ao dia } \\
\hline Até 2 & 171 & 28,9 & 162 & 25,5 & 333 & 27,1 \\
\hline Acima de 2 & 422 & 71,1 & 473 & 74,5 & 895 & 73,0 \\
\hline \multicolumn{7}{|c|}{ Número de vezes na semana e quantidade de horas ao dia que assiste à TV } \\
\hline Até 2 vezes e até 2 horas & 37 & 6,2 & 33 & 5,2 & 70 & 5,7 \\
\hline Até 2 vezes e acima de 2 horas & 26 & 4,3 & 22 & 3,4 & 48 & 3,9 \\
\hline Mais de 2 vezes e até 2 horas & 134 & 22,7 & 130 & 20,5 & 264 & 21,5 \\
\hline Mais de 2 vezes e acima de 2 horas & 396 & 66,8 & 450 & 70,9 & 846 & 68,9 \\
\hline \multicolumn{7}{|c|}{ Número de vezes que utiliza o computador na semana } \\
\hline Até 2 & 185 & 38,4 & 257 & 58,5 & 442 & 48,0 \\
\hline Mais de 2 & 296 & 61,5 & 182 & 41,4 & 478 & 52,0 \\
\hline \multicolumn{7}{|c|}{ Quantidade de horas que utiliza o computador ao dia } \\
\hline Até 2 & 280 & 58,2 & 338 & 76,9 & 618 & 67,1 \\
\hline Acima de 2 & 201 & 41,8 & 101 & 23,1 & 302 & 32,9 \\
\hline \multirow{2}{*}{\multicolumn{7}{|c|}{ Número de vezes na semana e quantidade de horas ao dia que utiliza o }} \\
\hline & \multicolumn{6}{|c|}{ computador } \\
\hline Até 2 vezes e até 2 horas & 174 & 36,2 & 244 & 55,5 & 418 & 45,4 \\
\hline Até 2 vezes e acima de 2 horas & 23 & 4,8 & 29 & 6,6 & 52 & 5,7 \\
\hline Mais de 2 vezes e até 2 horas & 93 & 19,3 & 65 & 14,8 & 158 & 17,2 \\
\hline Mais de 2 vezes e acima de 2 horas & 191 & 38,7 & 101 & 23,1 & 292 & 31,7 \\
\hline
\end{tabular}

\section{Discussão}

A ocorrência e características dos sintomas musculoesqueléticos na coluna lombar em escolares das escolas municipais de uma cidade do interior do Estado de São Paulo em 2007 mostraram que a prevalência de dor lombar foi de 19,5\%, sendo $7 \%$ nos meninos e $12,5 \%$ nas meninas; as meninas praticam natação, futebol e basquetebol, e os meninos, futebol e voleibol; que a maioria dos adolescentes passa mais de 2 vezes por semana e acima de 2 horas por dia utilizando computador ou TV, e os principais fatores associados com os sintomas na coluna lombar foram o gênero feminino, a quantidade de horas que assiste à TV ao dia e a prática de esportes.

Uma limitação do presente estudo, que necessita ser examinada, é o fato de que todos os 
Distribuição das frequências absoluta e relativa do sexo, faixa etária e prática de esportes fora da escola, segundo a presença da dor lombar e valores do teste estatístico.

\begin{tabular}{|c|c|c|c|c|c|c|c|}
\hline \multirow[t]{3}{*}{ Fatores de risco } & \multicolumn{4}{|c|}{ Dor lombar } & \multicolumn{2}{|c|}{ Total } & \multirow[t]{3}{*}{ Teste } \\
\hline & \multicolumn{2}{|c|}{ Não } & \multicolumn{2}{|c|}{ Sim } & \multirow[b]{2}{*}{$\mathbf{n}$} & \multirow[b]{2}{*}{$\%$} & \\
\hline & $\mathrm{n}$ & $\%$ & $\mathrm{n}$ & $\%$ & & & \\
\hline \multicolumn{8}{|l|}{ Sexo } \\
\hline Masculino & 510 & 51,2 & 86 & 35,6 & 596 & 48,4 & $\chi^{2}=18,8409$ \\
\hline Feminino & 485 & 48,7 & 155 & 64,4 & 640 & 51,6 & $p=0,000014$ \\
\hline \multicolumn{8}{|l|}{ Faixa etária (anos) } \\
\hline 11 & 169 & 16,9 & 58 & 24,1 & 227 & 18,4 & $\chi^{2}=12,5361$ \\
\hline 12 & 252 & 25,3 & 39 & 16,1 & 291 & 23,6 & $p=0,0057$ \\
\hline 13 & 271 & 27,2 & 71 & 29,5 & 342 & 27,6 & \\
\hline 14 & 303 & 30,6 & 73 & 30,3 & 376 & 30,4 & \\
\hline \multicolumn{8}{|c|}{ Prática de esportes fora da escola } \\
\hline Não & 579 & 58,1 & 85 & 35,2 & 664 & 53,7 & $\chi^{2}=40,9988$ \\
\hline Sim & 416 & 41,9 & 156 & 64,8 & 572 & 46,3 & $p=0,0001$ \\
\hline
\end{tabular}

mensuramentos foram baseados em autorrelatos. Devido à confidencialidade, não foram pesquisados, junto aos familiares, os dados relativos aos sintomas, práticas esportivas, frequência e horas na frente da TV/computador respondidos pelos escolares. Em alguns casos, pode ter ocorrido, principalmente sobre os sintomas, a dificuldade dos adolescentes em se lembrarem sobre a presença ou ausência dos mesmos após doze meses. Contudo, os apontamentos deste estudo são comparáveis aos da literatura específica.

Os resultados do presente estudo mostram que a prevalência de dores lombares (19,5\%) é, em geral, semelhante às taxas registradas na literatura, as quais variam entre $13 \%$ e $64 \%$. Nos estudantes de uma região do Kuwait 16, foi observado que $50,8 \%$ dos meninos e $64,7 \%$ das meninas relataram essa queixa, e, na Turquia, constataram que estava presente em $40,9 \%$ da população estudada 17. Em 204 adolescentes de Moçambique, foi notado que $13,5 \%$ referiram dor 18 . Alguns estudos verificaram que $28,4 \%$, $26 \%$ e $34 \%$ dos escolares relataram dor na coluna, respectivamente 19,20,21.

Essas variações nos relatos de dor podem ser relacionadas à definição de dor lombar, às diferenças entre populações, ao fator tempo, aos fatores psicológicos, como também a outros aspectos da personalidade das crianças.

Em relação ao gênero, pode-se notar que as meninas tiveram cerca de duas vezes mais chances de ter dor lombar do que os meninos. Os estudos 22,23,24 apontaram que as meninas apresentaram duas vezes mais chances de possuir dor em relação aos meninos. Nos adolescentes dinamarqueses 24 , o risco foi $15 \%$ maior, e, nos escolares do Kuwait 25, as meninas apresentaram cerca de $40 \%$ maior risco para o desenvolvimento de dor em comparação aos meninos.

As diferenças entre os sexos podem ser explicadas por meio de algumas suposições. A primeira está relacionada à força física, que é menor nas mulheres do que nos homens, fazendo com que o gasto energético delas seja maior quando expostas à demanda de trabalho similar, aumentando o risco de sobrecarga musculoesquelética. Uma outra é de ordem psicossocial, pois se acredita que as mulheres se queixam com maior frequência do que os homens, ou seja, de acordo com essa linha, os contrastes resultam das diferenças na predisposição de homens e mulheres quanto a relatar as informações. Talvez as mulheres tenham mais permissão social para falarem sobre seus sintomas e sentimentos ou tenham uma capacidade de observação mais desenvolvida, devendo-se ambos os eventos a fatores sociais e educacionais 26 .

Os esportes mais praticados fora da escola pelas meninas são a natação, o futebol e o basquetebol e, pelos meninos, são o futebol e o voleibol. Em escolares do Município de Niterói (Rio de Janeiro), os esportes mais praticados pelos meninos foram o futebol e a corrida, enquanto que, pelas meninas, foram a corrida e a dança 27 . Na Noruega, os esportes mais praticados foram o handebol e o futebol 28. Uma possível explicação para tal fato está relacionada aos fatores culturais, no caso específico do futebol, enquanto as outras 
Tabela 3

Distribuição das frequências absoluta e relativa das atividades sedentárias, segundo a presença da dor lombar e valores do teste estatístico.

\begin{tabular}{|c|c|c|c|c|c|c|c|}
\hline \multirow[t]{3}{*}{ Fatores } & \multicolumn{4}{|c|}{ Dor lombar } & \multicolumn{2}{|c|}{ Total } & \multirow[t]{3}{*}{ Teste } \\
\hline & \multicolumn{2}{|c|}{ Não } & \multicolumn{2}{|c|}{ Sim } & \multirow[b]{2}{*}{$\mathbf{n}$} & \multirow[b]{2}{*}{$\%$} & \\
\hline & $\mathrm{n}$ & $\%$ & $\mathbf{n}$ & $\%$ & & & \\
\hline \multicolumn{8}{|l|}{ Uso da TV } \\
\hline Não & 7 & 0,8 & 1 & 0,4 & 8 & 0,6 & $\chi^{2}=0,25125$ \\
\hline Sim & 988 & 99,2 & 240 & 99,6 & 1.228 & 99,4 & $p=0,6162$ \\
\hline \multicolumn{8}{|c|}{ Número de vezes que assiste à TV na semana } \\
\hline Até 2 & 100 & 10,0 & 17 & 7,1 & 117 & 9,5 & $\chi^{2}=2,0074$ \\
\hline Mais de 2 & 889 & 90,0 & 222 & 92,9 & 1.111 & 90,5 & $p=0,1565$ \\
\hline \multicolumn{8}{|c|}{ Quantidade de horas que assiste à TV ao dia } \\
\hline Até 2 & 292 & 29,3 & 41 & 17,0 & 333 & 27,1 & $\chi^{2}=14,6115$ \\
\hline Acima de 2 & 698 & 70,6 & 197 & 82,0 & 895 & 72,9 & $p=0,0001$ \\
\hline \multicolumn{8}{|c|}{ Número de vezes na semana e quantidade de horas ao dia } \\
\hline \multicolumn{8}{|c|}{ que assiste à TV } \\
\hline Até 2 vezes e até 2 horas & 61 & 6,1 & 9 & 3,7 & 70 & 5,7 & $\chi^{2}=16,3079$ \\
\hline Até 2 vezes e acima de 2 horas & 41 & 4,1 & 7 & 2,9 & 48 & 3,9 & $p=0,0009$ \\
\hline Mais de 2 vezes e até 2 horas & 232 & 23,3 & 32 & 13,0 & 264 & 24,5 & \\
\hline Mais de 2 vezes e acima de 2 horas & 657 & 66,5 & 189 & 78,4 & 846 & 68,9 & \\
\hline \multicolumn{8}{|l|}{ Uso do computador } \\
\hline Não & 254 & 25,5 & 62 & 25,7 & 316 & 25,5 & $\chi^{2}=0,00402$ \\
\hline Sim & 741 & 74,5 & 179 & 74,3 & 920 & 74,5 & $p=0,9495$ \\
\hline \multicolumn{8}{|c|}{ Número de vezes que utiliza o computador na semana } \\
\hline Até 2 & 350 & 47,4 & 92 & 50,5 & 442 & 48,0 & $\chi^{2}=0,5708$ \\
\hline Mais de 2 & 388 & 52,6 & 90 & 49,5 & 478 & 52,0 & $p=0,4499$ \\
\hline \multicolumn{8}{|c|}{ Quantidade de horas que utiliza o computador ao dia } \\
\hline Até 2 & 507 & 68,0 & 111 & 63,4 & 618 & 67,2 & $\chi^{2}=1,3748$ \\
\hline Acima de 2 & 238 & 32,0 & 64 & 36,5 & 302 & 32,8 & $p=0,2409$ \\
\hline \multicolumn{8}{|c|}{ Número de vezes na semana e quantidade de horas ao dia } \\
\hline \multicolumn{8}{|l|}{ que utiliza o computador } \\
\hline Até 2 vezes e até 2 horas & 320 & 63,4 & 98 & 49,0 & 418 & 45,4 & $\chi^{2}=5,8638$ \\
\hline Até 2 vezes e acima de 2 horas & 36 & 7,2 & 16 & 8,0 & 52 & 5,7 & $p=0,1184$ \\
\hline Mais de 2 vezes e até 2 horas & 124 & 24,7 & 34 & 17,0 & 158 & 17,2 & \\
\hline Mais de 2 vezes e acima de 2 horas & 240 & 46,7 & 52 & 26,0 & 292 & 31,7 & \\
\hline
\end{tabular}

Tabela 4

Resultado da análise multivariada de regressão logística. Modelo final para associações independentes com a presença de sintomatologia dolorosa.

\begin{tabular}{|c|c|c|c|c|}
\hline Variáveis & Referência & Valor de $\mathrm{p}$ & OR ajustado & IC95\% \\
\hline \multirow[t]{2}{*}{ Sexo } & Feminino & 0,0001 & 1,93 & $1,43-2,60$ \\
\hline & Masculino & & & \\
\hline \multirow[t]{2}{*}{ Prática de esportes fora da escola } & Sim & 0,0001 & 2,58 & $1,92-3,48$ \\
\hline & Não & & & \\
\hline \multirow[t]{2}{*}{ Quantidade de horas que assiste à TV ao dia } & Acima de 2 & 0,01 & 1,86 & $1,29-2,69$ \\
\hline & Até 2 & & & \\
\hline
\end{tabular}


modalidades são populares entre estudantes de diferentes nacionalidades 29 .

Como mencionado anteriormente, verificou-se a maior frequência de escolares utilizando a TV mais de 2 vezes por semana e acima de 2 horas por dia, e, quanto ao computador/ videogame, o maior número de meninos acima de 2 horas ao dia e as meninas até 2 horas ao dia. Adolescentes noruegueses e dinamarqueses que assistem à TV e utilizam o computador por 3 horas diárias relataram maior prevalência de dor 4,28, enquanto que, nos escolares finlandeses, foi notada maior frequência de dor nos que utilizam o computador e assistem à TV de 2 a 3 horas por dia 30 .

No presente estudo, a análise multivariada mostrou que o gênero feminino, quantidade de horas que assiste à TV ao dia e a prática de esportes são importantes preditores de dores na coluna lombar.

Os escolares que permaneciam em frente à TV acima de duas horas apresentaram quase duas vezes mais chances de possuir dor. Nos escolares que permaneciam por mais de duas horas, o risco de dor lombar foi de 1,27 (1,0-1,52) 1, e, em escolares noruegueses 28 e os de uma região do Kwuait 31 , a taxa de risco foi $30 \%$ maior nos que permanecem mais de duas horas na frente da TV. Outros estudos não apontaram relação entre as mesmas 24,30.

Essa associação pode ser devido ao tempo prolongado na posição sentada e/ou posturas incorretas, mobiliário inadequado e mal organizado e/ou sedentarismo ${ }^{1}$. A postura sentada gera várias alterações nas estruturas musculoesqueléticas dos diversos segmentos corporais. Aumenta em, aproximadamente, $35 \%$ a pressão interna no núcleo do disco intervertebral, estira todas as estruturas (ligamentos, pequenas articulações e nervos) da coluna vertebral, reduz a circulação de retorno dos membros inferiores e promove o desenvolvimento de processos inflamatórios nas estruturas osteomusculares com sintomatologia dolorosa associada 32 .

Os escolares que praticam esportes fora da escola têm duas vezes e meia mais chances de possuir dor lombar. Estudos 13,17,31 indicaram associação significativa entre as práticas esportivas e dor lombar, permitindo, dessa forma, que os autores afirmassem que as atividades esportivas podem ser fator de risco para o surgimento desses sintomas na infância e na adolescência.

Tal fato pode ser justificado por um conjunto de fatores, tais como o tipo de esporte, o nível de exigência, a intensidade do treinamento e o grau de trauma agudo gerado pelo esporte, principalmente em crianças e adolescentes 13,33,34. Em contraposição, alguns pesquisadores têm mostrado que a participação em esportes não apresenta correlação com a prevalência de dores na coluna 21,22,28,35. Nessa linha de raciocínio, os autores consideram que a participação esportiva regular é protetora para o surgimento de sintomas musculoesqueléticos pelo fato de provocar mudanças fisiológicas nas estruturas musculares, aumentar a capacidade de suportar carga estática e repetitiva e pelos efeitos secundários (melhora do humor, autoeficácia, equilíbrio e habilidades).

As demais variáveis (uso da TV e do computador/videogame e quantidade de horas que assiste à TV ao dia ou usa o computador/videogame) não apresentaram resultados estatisticamente significantes. No entanto, a literatura enquadra essas variáveis como fatores de risco para a presença de sintomas em escolares.

Dessa maneira, os resultados do presente estudo mostraram que há predomínio da manifestação dos sintomas musculoesqueléticos (dor lombar) na coluna vertebral nas adolescentes e que há associação entre os sintomas e o sexo, a quantidade de horas que assiste à TV ao dia e a prática de esportes. Um ponto extremamente favorável deste estudo foi o número de indivíduos questionados com o mesmo nível socioeconômico, e uma contribuição importante é que dados dessa natureza e de outras que dela decorrerem possam melhorar a compreensão das relações entre as variáveis e oferecer elementos úteis ao planejamento de medidas visando à manutenção, à melhora e à promoção do bem-estar físico e psicológico dos estudantes. 


\section{Resumo}

O objetivo foi verificar a prevalência de dor lombar em adolescentes e sua relação com a prática de esportes $e$ atividades sedentárias. Realizou-se um estudo transversal com 1.236 escolares das $5 \underline{a}$ a $8 \underline{a}$ séries do Ensino Fundamental de Bauru, São Paulo, Brasil. Foram utilizados um protocolo estruturado e o questionário nórdico de sintomas musculoesqueléticos. A análise foi realizada mediante uma abordagem descritiva, bivariada e multivariada por regressão logística binária. Notou-se que a prevalência de dor lombar foi de 19,5\%, sendo $7 \%$ nos meninos e $12,5 \%$ nas meninas, com diferença estatisticamente significante entre os gêneros $(p<0,00001)$, a faixa etária $(p=0,0057)$ e prática de esportes $(p=0,0001)$. Na análise bivariada e multivariada, observaram-se associações independentes entre dor lombar e o gênero feminino, horas na frente da TV e prática de esportes. A dor lombar em escolares pode ter consequências importantes para dores crônicas em adultos, e a compreensão das relações entre as variáveis oferecerá elementos úteis de medidas visando à manutenção, à melhora e à promoção do bem-estar dos estudantes.

Dor Lombar; Estudantes; Adolescente; Fatores de Risco; Prevalência

\section{Referências}

1. Balagué F, Dutoit G, Waldburg M. Low back pain in schoolchildren - an epidemiological study. Scand J Rehabil Med 1988; 20:175-9.

2. Méndez FJ, Gómez-Coneza A. Postural hygiene program to prevent low back pain. Spine 2001; 26:1280-6.

3. Burton K. Low back pain in children and adolescents: to treat or not. Bull Hosp Jt Dis 1996; 3:127-9.

4. Harreby M, Nygaad B, Jenssen T, Larsen E, StorrPaulsen A, Lindahl A, et al. Risk factors for low back pain in a cohort of 1389 danish school children: an epidemiologic study. Eur Spine J 1999; 8:444-50.

5. Wedderkopp N, Leboeuf-Yde C, Andersen LB, Karsten F, Hansen HS. Back pain reporting pattern in a Danish population-based sample of children and adolescents. Spine 2001; 26:1879-83.

6. Wedderkopp N, Andersen LB, Karsten F, LeboeufYde C. Back pain reporting in young girls appears to be puberty-related. BMC Musculoskelet Disord 2005; 6:1-5.

\section{Colaboradores}

A. De Vitta contribuiu com a pesquisa, análise e interpretação dos dados e redação final. M. G. Martinez contribuiu com a pesquisa, análise e interpretação dos dados. N. T. Piza contribuiu com a pesquisa, análise e interpretação dos dados. S. F. A. P. Simeão contribuiu com a análise estatística e revisão crítica. N. P. Ferreira contribuiu com a coleta e interpretação dos dados.

\section{Agradecimentos}

À Fundação de Amparo à Pesquisa do Estado de São Paulo (FAPESP; 08/56306-2).
7. Cardon G, Balagué F. Low back pain prevention's effects in schoolchildren: what is the evidence? Eur Spine J 2004; 13:663-79.

8. Sheir-Neiss GI, Kruse RW, Rahman T, Jacobson LP, Pelli JA. The association of backpack use and back pain in adolescents. Spine 2003; 28:922-30.

9. Gunzburg R, Balagué F, Nordin M, Szpalski M, Duyck D, Bull D, et al. Low back pain in a population of school children. Eur Spine J 1999; 8:439-43.

10. Kovacs FM, Gestoso M, Del Real MTG, Lopez J, Mufragg N, Mendez JE. Risk factors for non-specific low back pain in schoolchildren and their parents: a population based study. Pain 2003; 103:259-68.

11. Korovessis P, Koureas GK Papazisis Z. Correlation between backpack weight and way of carrying, sagittal and frontal spinal curvatures, athletic activity, and dorsal and low back pain in schoolchildren and adolescents. J Spinal Disord Tech 2004; 17:33-40. 
12. Barros E. Cross-cultural adaptation of the Nordic musculoskeletal questionnaire. Int Nurs Rev 2003; 50:101-8.

13. Sato T, Ito T, Hirano T, Morita O, Kikuchi R, Endo N, et al. Low back pain in childhood and adolescence: a cross-sectional study in Niigata City. Eur Spine J 2008; 17:1441-7.

14. Norman GR, Streiner DL. Biostatitics: the bare essentials. St. Louis: Mosby; 1994.

15. Zapata AL, Moraes PAJ, Leone C, Doria UF, Silva AAC. Pain and musculoskeletal pain syndromes related to computer and videogame use in adolescents. Eur J Pediatr 2006; 165:408-14.

16. Shehab DK, Al-Jarallah KF, Al-Ghareeb F, Sanaseeri $\mathrm{S}$, Al-Fadhli M, Habeeb S. Is low back pain prevalent among Kuwaiti children and adolescents? A governorate based study. Med Princ Pract 2004; 13:142-6.

17. Çakmak AMD, Yucel BMD, Ozyalçn SN, Bayraktar MD, Bulent MD, Ibrahim U, et al. The frequency and associated factors of low back pain among a younger population in Turkey. Spine 2004; 29:1567-72.

18. Prista A, Balagué F, Nordin M, Skovron ML. Low back pain in Mozambican adolescents. Eur Spine J 2004; 13:341-5.

19. Bejia I, Abid N, Ben Salem K, Letaief M, Younes M, Touzi M, et al. Low back pain in a cohort of 622 Tunisian schoolchildren and adolescents: an epidemiological study. Eur Spine J 2006; 15:2-7.

20. Hestbaek L, Leboeuf-Yde C, Kyvik KO. Is comorbidity in adolescence a predictor for adult low back pain? A prospective study of a young population. BMC Musculoskelet Disord 2006; 7:29.

21. Chiang HY, Jacobs K, Orsmond G. Gender-age environmental associates of middle school students low back pain. Work 2006; 26:197-206.

22. Troussier B, Davoine B, Gaudemaris R, Foconni J, Phelip X. Back pain in school children: a study among 1178 people. Scand J Rehabil Med 1994; 26:143-6.

23. Feldman DE, Rossignol M, Shrier L, Abenhaim L. Smoking: a risk factors for development of low back pain in adolescents. Spine 1999; 24:2492-6.

24. Diepenmaat MF, Wal HCW, Hirasing R. A neck/ shoulder, low back, and arm pain in relation to computer use, physical activity, stress, and depression among Dutch adolescents. Pediatrics 2006; 5:412.
25. El-Metwally A, Salminen JJ, Auvinen A, Macfarlane G, Mikkelsson M. Risk factors for development of non-specific musculoskeletal pain in preteens and early adolescents: a prospective 1-year follow-up study. BMC Musculoskelet Disord 2007, 8:1-8.

26. De Vitta A, Zapater AR, Campos RS, Padovani CR. Desconfortos musculoesqueléticos percebidos em trabalhadores de diferentes faixas etárias, gêneros e ocupações. Fisioterapia em Movimento 2007; 20:29-35.

27. Silva RCR, Malina RM. Nível de atividade física em adolescentes do Município de Niterói, Rio de Janeiro, Brasil. Cad Saúde Pública 2000; 16:1091-7.

28. Sjolie NA. Low-back pain in adolescents is associated with poor hip mobility and high body mass index. Scand J Med Sci Sports 2004; 14:168-75.

29. Myers L, Strikmiller PK, Webber LS, Berenson GS. Physical and sedentary activities in school children grades 5-8: the Bogalusa Heart Study. Med Sci Sports Exerc 1996; 28:852-9.

30. Hakala P, Rimpelä A, Salminen JJ, Virtanen SM, Rimpelä M. Back, neck, and shoulder pain in Finnish adolescents: national cross sectional surveys BMJ 2002; 325:1-4.

31. Shehab DK, Al-Jarallah KF. Nonspecific low-back pain in Kuwaiti children and adolescents: associated factors. J Adolesc Health 2005; 36:32-5.

32. Zapater AR, Silveira DM, De Vitta A, Padovani CR, Silva JPC. Postura Sentada: a eficácia de um programa de educação para escolares. Ciênc Saúde Coletiva 2004; 9:191-9.

33. Kujala UM, Taimela S, Erkintalo M, Salminen JJ, Kaprio J. Low back pain in adolescent athletes. Med Sci Sports Exerc 1996; 28:165-70.

34. Andersen LB, Wedderkopp N, Leboeuf-Yde C. Association between back pain and physical fitness in adolescents. Spine 2006; 31:1740-4.

35. Balague F, Troussier B, Salminen JJ. Non-specific low back pain in children and adolescents: risk factors. Eur Spine J 1999; 8:429-38.

Recebido em 15/Out/2010

Versão final reapresentada em 15/Abr/2011 Aprovado em 06/Mai/2011 